\title{
NOTE DI TOPONOMASTICA ITALIANA. I
}

Nella Prefazione ad un importante contributo negli studii sulla toponomastica italiana $^{1}$, pubblicato del Dipartimento di Linguistica dell'Università di Firenze, Carlo Alberto Mastrelli scriveva: "L'Italia non si è ancora data un 'Istituto Italiano di Onomastica', nonostante i voti piú volte espressi da almeno cento anni a questa parte. Ma all'indifferenza e all'insensibilità di uno stato che non ha avvertito la necessità di provvedere alla raccolta completa dei 'nomi di luogo' e dei 'nomi di persona' per assicurare un servizio di informazione e di orientamento per la società contemporanea, non poteva corrispondere una pari indifferenza e insensibilità da parte del mondo universitario, che vede in una impresa del genere una fonte inesauribile di dati rilevanti e preziosi anche per le loro implicazioni scientifiche"2.

$\mathrm{E}$, infatti, per l'antroponimia ha dato un consistente contributo Emidio De Felice ${ }^{3}$, già dell'Università di Genova, mentre per opera di un gruppo di specialisti di varie Università - Genova, Padova, Torino, Udine - è stata recentemente pubblicata la prima sum$m a$ toponimica italiana ${ }^{4}$. Essa raccoglie, in grande formato e bella veste tipografica, $\mathrm{i}$ nomi di tutti i comuni e quelli (principali) di regioni, mari, laghi, fiumi, monti, isole d'Italia, accompagnati da precise indicazioni geografiche, cenni essenziali sulle singolarità del paese e sugli eventuali mutamenti del nome e, sopra tutto, dall'indicazione delle fonti, dalla discussione dell' etimologia e da riferimenti bibliografici ${ }^{5}$, che sono poi - questi ultimi - i dati che conferiscono spessore e validità scientifica all'opera ${ }^{6}$.

1 F. GRANUCCI, Prontuario bibliografico di toponomastica italiana, con prefazione di C.A. MASTRELLI, Firenze, 1988.

2 C.A. MASTRELLI, Prefazione, in F. GRANUCCI, $O$ p.cit, p. non numerata.

3 E. DE FELICE, Dizionario dei cognomi italiani, Milano, 1978; I cognomi italiani. Rilevamenti quantitativi dagli elenchi telefonici: informazioni socioeconomiche e culturali, onomastiche $e$ linguistiche, Bologna, 1980; I nomi degli Italiani. Informazioni onomastiche e linguistiche, socioculturali e religiose. Rilevamenti quantitativi dei nomi personali dagli elenchi telefonici, Venezia, 1982; Dizionario dei nomi italiani. Origine, etimologia, storia, diffusione e frequenza di oltre 18.000 nomi, Milano, 1986.

4 G. GASCA QUEIRAZZA - C. MARCATO - G.B. PELLEGRINI - G. PETRACCO SICARDI A. ROSSEBASTIANO, Dizionario di toponomastica. Storia e significato dei nomi geografici italiani, Torino, 1990.

5 Questi si trovano sia all'interno delle singole voci, sia nella lista delle Abbreviazioni Bibliografiche, che a dispetto del titolo ha lo spessore (pp. XIII-XXVII) di una vera e propria bibliografia. Nel nostro sondaggio - s.u.Gamalero - abbiamo, però, incontrato l'abbreviazione di un lavoro (Passaggio 1963) che non è registrato e che purtroppo non compare neppure in F. GRANUCCI, Op.cit.

Gli elementi che abbiamo elencato, partecipano tutti a formare il corpo della voce. Abbiamo notato, 
I meriti di quest'impresa, che resterà nella storia della ricerca toponomastica, vanno attribuiti “anzitutto alla principale collaboratrice [Carla Marcato]" e agli altri "eccellenti specialisti delle regioni di cui si sono occupati: Giulia Petracco Sicardi che ha elaborato i lemmi liguri. Giuliano Gasca Queirazza e Alda Rossebastiano che hanno rivolto le loro cure alla sezione piemontese" 7 , mentre Giovan Battista Pellegrini "si è riservato di redigere la parte che si riferisce a due regioni: 1'Alto Adige e la Sicilia",

Tipica opera di consultazione, il Dizionario di toponomastica potrà essere utilizzato da tutti, ma non letto tutto di seguito ${ }^{9}$. Le sue settecentocinquanta pagine saranno invece scandagliate secondo le esigenze di ciascuno: dagli studiosi, per la messe dei dati scientifici prudentemente ma sapientemente valiati; dagli altri, per soddisfare spesso una semplice ma comprensibile curiosità.

“La toponomastica troppe volte lascia aperti i problemi o ne pone di piú di quanti ne possa risolvere" 10 , per questo le osservazioni che seguono vanno considerate delle semplici note di lettura, scaturite da un primo sondaggio, operato senz'altro criterio che quello di 'andare a vedere' quale proposta era stata accolta, per quei toponimi di cui abbiamo qualche conoscenza.

\section{PALERMO}

A Giovan Battista Pellegrini, che ha redatto la voce, "non pare destituita di fon-

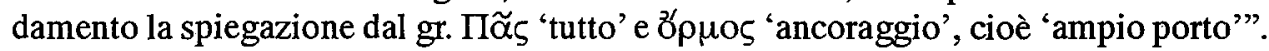
A noi sembra, francamente, una prudenza eccessiva, perché il nome è sicuramente greco $^{11}$; infatti, Gerhard Rohlfs ${ }^{12}$ lo cita come esempio ${ }^{13}$ del predominio di questa lingua in Sicilia fin dai tempi dei Cartaginesi ${ }^{14}$. Secondo lo studioso tedesco la forma

però, che quelle relative al Piemonte sono arricchite da un dato esclusivo: le citazioni di passi di poesie - spesso preziose - in cui compare il toponimo. Noi non sappiamo spiegare tale disparità. E' certamente da rifiutare (lectiofacilior) che sia dovuta al fatto che la sede della casa editrice è a Torino. Si può forse pensare (lectio difficilior) che sia il segno distintivo di una formazione filologica dell'Autrice.

7 Per la verità Giuliano Gasca Queirazza non ha firmato che una trentina di agiotoponimi; per contro un certo numero di toponimi di area alessandrina, porta la firma di Elena Papa.

8 G.B. PELLEGRINI, Presentazione, in G. GASCA QUEIRAZZA et alii, $O p$. cit., p.VIII.

9 Una simile lettura, mirata cioè a cogliere le vicende storiche dell'Italia attraverso il dato toponimico, può essere fatta per il volume: G.B. PELLEGRINI, Toponomastica italiana. 10.000 nomi di città, paesi, frazioni, regioni, contrade, fiumi, monti spiegati nella loro origine e storia, Milano, 1990.

10 T. BOLELLI, Schiavi-Liberi una sola città, "La Stampa", 12.03.1991.

11 Ad 'ampio porto', altri preferiscono 'porto completamente sicuro' (cfr. G. ROHLFS, Historische Sprachschichten im modernen Sizilien, München, 1975, p.12; G. BONFANTE, Rec. $a$ G. Rohlfs, Op. cit., "Paideia", XXXI/3-4 [1976], p. 210 [= Scritti Scelti di G. Bonfante, a cura di R. GENDRE, Alessandria, 1986-1992. III. Siciliano, 1992, p.221]).

12 G. ROHLFS, Op.cit., p. 11.

13 Insieme a Trapaniche deriva dal greco dialettale, ma certamente antico $\delta \rho \alpha \pi \alpha v o \varsigma$ (attico $\delta \rho \varepsilon \Pi \alpha v o \zeta)$ 'falce'; come si legge anche in G.B. PELLEGRINI, s.u. Trapani. 


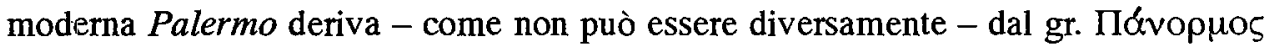
attraverso il latino Palermus, che presenta la dissimilazione non rara di $n . . . r$ a $l . . . r$ e il passaggio ŏ > $\breve{e}^{15}$. Poiché le prime attestazioni della forma moderna si hanno in arabo, Giovan Battista Pellegrini pensa invece che "Panormus sia stato reso con Balermus soprattutto in bocca degli Arabi, cioè con balarm ma pronunciato, con la nota imâla, balerm"16.

\section{TERAMO/TERNI}

L'odierna Teramo era l'antica Interamna Praetutiōrum (citata s.u. Terni senza l'indicazione della quantità), mentre Terni era l'Interamna Nārs (Nahars). Entrambe, infatti, sorgono alla confluenza di due fiumi (inter amnes): il Vezzola con il Tordino, quella sul cui territorio erano stanziati i Praetūt $\bar{\imath}$; il Serra con il Nera (Närs/Nahars) l'altra. Non c'è dubbio, dunque, che i due toponimi odierni derivino da Interamna ${ }^{17}$, con accentazione proparossitona, che si spiega con la positio debilis della penultima breve (Intérămna). Per questo, s.u. Terni (ma meglio sarebbe stato s.u. Teramo), si doveva evitare di considerare quasi come alternativa l'ipotesi secondo cui "Interamna [...] è un adattamento latino di una voce italica che ha riscontro nell'umbro (tremnu 'tabernāculō') e nel greco ( $\{\hat{\varepsilon} \rho \alpha \mu \nu \alpha, \tau \varepsilon \rho \rho \mu \nu \alpha$ n.pl. 'case, abitazioni') [...] tratto dal tema che appare nell'osco tríibúm 'domum' [...], considerando in- (da *en-) come la preposizione agglutinata"18. Bene ha fatto, invece, l'Autrice ad ignorare la proposta di Emesto Giammarco ${ }^{19}$, che ipotizza per Teramo una fase di mediazione 'sabina' continuata anche dopo la latinizzazione dell'italico e documentata dall'accento proparossitono, attraverso le fasi ${ }^{*}$ Trebnu $>{ }^{*}$ Tremnu $>$ Teramum, già lapidariamente liquidata da Giuliano Bonfante come "assolutamente indefendibile" 20 .

\section{ANGRI}

D'accordo con Giovanni Alessio ${ }^{21}$, l'Autrice della voce prende le distanze dalla posizione di Gerhard Rohlfs ${ }^{22}$, che considera il nome di origine greca ${ }^{23}$ e lo fa deri-

14 Infatti Palermo, pur essendo uno dei grandi cent ri d'irradiazione cartaginese, ha un nome greco; cosí Trapani, che si trovava in pieno territorio cartaginese.

15 Cfr., per esempio, il latino hospes < *hósti-potis.

16 G.B. PELLEGRINI, Toponomastica cit., p. 296.

17 Per Terni, naturaimente si deve postulare un passagio ad Interamnia.

18 G. ALESSIO-M. de GIOVANNI, Preistoria e protostoria linguistica dell'Abruzzo, Lanciano, 1983, p. 27.

19 E. GIAMMARCO, Per la storia linguistica di Interamna e di Teate, "Abruzzo" 21 (1983), pp. 161-168.

20 G. BONFANTE, Il nome di 'Tèramo', "Lincei - Rendiconti", XLII/5-6 (1987) (ma 1988), p. 91.

21 G. ALESSIO, Panorama di toponomastica italiana, Napoli, 1958, p. 80.

22 G. ROHLFS, Lexikon graecanicum Italiae inferioris. Etymologisches Wörterbuch der 
vare invece "dal latino ancrae 'convalles' da cui anche il calabrese dialettale angra 'pezzo di terra coltivata vicino al fiume', il siciliano angru 'rupe' e nella toponomastica calabrese Angra, Angri, ecc."24. Come si vede, non si fa cenno ad una tutt'altro che improbabile derivazione di Angri dal nome degli Angrivari ${ }^{25}$. Poiché essi sono una delle tribú germaniche che, dopo varie vicende, contribuiscono a formare la lega dei Sassoni, è logico pensare che tra i ventimila Sassoni, che secondo Paolo Diacono ${ }^{26}$ vengono in Italia al seguito di Alboino, ci sia anche un contingente di Angrari o An$\operatorname{gri}^{27}$, come ormai si chiamano ${ }^{28}$. E'ben vero che dopo qualche anno questi se ne tornano delusi al di là delle Alpi, ma la toponomastica centro-meridionale testimonia che schiere piú o meno numerose di Sassoni sono rimaste in quelle regioni ${ }^{29}$. Di conseguenza, si può ipotizzare che tra di esse non mancassero gruppi di Angri, che avrebbero cosí lasciato traccia della loro presenza nel toponimo Angri $^{30}$.

\section{AOSTA}

Che "l'odierna Aosta" non sia "che la continuazione di Augusta"31 ci sembra almeno una formulazione imprecisa. La forma italiana Aosta, confermata dalla forma francese Aoste, presuppone un antecedente diretto *Agusta, non diversamente da Ascoli, che correttamente ${ }^{32}$ è fatto derivare da Ausculum ma attraverso una forma intermedia *Asculum, con la riduzione del dittongo $a u$ ad $a$.

unteritalienischen Gräzität, Tübingen, 1964.

$\mathrm{Vi}$ vede, infatti, il greco $\alpha \gamma \kappa \varepsilon \alpha, \alpha \gamma \kappa \rho \varepsilon \alpha$.

C. MARCATO, s.u.

Il nome di questa popolazione, che intorno al sec. I d. C. è stanziata sul corso inferiore del Weser, deriva dal germanico *angria 'spiazzo erboso' (cfr. alto tedesco antico angar 's.s.', nordico antico angr anche 'baia') e dal suffisso - wari. Gli Angrivari, dunque, sono gli 'abitanti delle praterie'.

26 Historia Longobardorum, II, 6.

27 Intorno al 97 d.C., gli Angrivari, costretti ad abbandonare le loro sedi sotto la pressione dei Cauci, si uniscono ai Camavi e, sconfitti i Bructeri, ne occupano il territorio. Piú tardi, in un'area un po'piú meridionale di quella da loro occupata, compaiono nella storia gli Angrari o Angri. Questa coincidenza - la scomparsa dei primi e la comparsa dei secondi pressoché nello stesso tempo e nella stessa area - fa pensare che debba trattarsi dello stesso popolo. Se cosí è, il nuovo nome non sarebbe altro che l'esito delle contrazioni o delle sincopi che una parola polisillabica germanica sempre subisce a causa dell'accento intensivo protosillabico.

28 Già nel libro XIII del Laterculum Ueronensis (sec. IV), l'elenco delle "gentes barbarae quae pullulaverunt sub imperatoribus", registra gli “Angri-Angriuari”.

29 Cfr. Sassinoro (BN), contrada Sassonia (sia presso Castrovillari, CS; sia presso Farindola, PE), podere Sassogna (presso Orvieto) riportati in F. SABATINI, Riflessi linguistici della dominazione longobarda nell'Italia mediana e meridionale, Firenze, 1963, p. 46.

30 Ulteriori notizie si trovano in R. DELPEZZO, E' 'Angri' un toponimo germanico?, “AION-Filologia Germanica”, XXII (1979), pp. 333-340.

31 C. MARCATO, s.u. Aosta.

32 C. MARCATO, s.u. Ascoli. 


\section{SESTO CALENDE}

“La specificazione Calende, già nota nell'anno 1240 'Sexto Kalendarum' [...] pare alluda al periodo in cui, fin da epoca antica, si teneva il mercato; dimenticata per un certo tempo o sostituita da Mercatum ricompare poi alla metà del sec. XIII"33. Queste notazioni richiedono qualche precisazione. Intanto il toponimo, nella forma Sexto Mercado, è già registrato quattro secoli prima, nell' anno $892^{34}$. Inoltre Sextus Calendarum, poi Sextus Calendas, allude sicuramente al mercato che si teneva ogni mese, allineandosi cosí ad altri tipi toponimici, quali lat. Forum ${ }^{35}$, lat. crist. Fera ${ }^{36}$, ill. $\mathrm{Terg}^{37}$ che fanno tutti riferimento a particolari condizioni di mercato.

\section{TRIESTE/ODERZO}

In una voce ${ }^{38}$ si legge che "il toponimo [scil. Tergeste] è stato interpretato come un derivato dalla base *terg- con il significato di 'mercato' [...] attribuito dapprima all'illirico, poi al venetico, ma è ipotesi discutibile". Nell'altra ${ }^{39}$, sempre a proposito di * terg- (in Opitergium) si dice che "non è assolutamente certo" che abbia il significato di 'mercato' e che "non è ascrivibile con sicurezza" al venetico.

Ma è una prudenza - quella sul significato del tema - che ancora una volta non ci sentiamo di condividere.

Non c'è dubbio, infatti, che sulla base sia del confronto con lo sl.ant. trŭg 'mercato' e con l'alb. treg 'mercato', sia dell'interpretazione "assai verosimile", secondo Giovan Battista Pellegrini ${ }^{40}$, del Negotiator dell' iscrizione di Scarbantia ${ }^{41}$, come resa tautologica dell'indigeno Tergitio, che il tema *terg- voglia dire 'mercato', commercio" ${ }^{42}$.

33 C. MARCATO, s.u. Sesto Calende.

34 Cfr. Codex Diplomaticus Longobardiae, in MHP, XIV, Torino, 1873.

35 'Mercato'. Cfr. C. MARCATO, s.uu. Forli (< Forum Livii); Forlimpopoli (< Forum Popilii); Fossombrone (< Forum Sempronii); Fornovo (< Forum novum). A proposito di quest'ultimo toponimo, l'Autrice fa una distinzione tra Fornovo di Taro e Fornovo San Giovanni (v. s. uu.). Per entrambi, infatti, è correttamente accol ta la derivazione da forum novum; ma, mentre per il secondo "il toponimo pare essere [...]"mercato nuovo", per il primo registra anche il significato di "forno nuovo' sulla base della denominazione dialettale fornov, dando cosí piú credito di quanto meriti alla proposta di Dante Olivieri (D. OLIVIERI, Nuova serie di appuntitoponomastici emiliano-romagnoli, in Ioanni Dominico Serra ex munere laeto inferiae. Raccolta di studi linguistici in onore di Giandomenico Serra, Napoli, 1959, p. 294).

36 'Grande mercato in occasione di feste religiose'. Cfr. C. MARCATO, s.u. Fiera di Primiero.

37 'Mercato, Commercio'. V. infra.

38 C. MARCATO, s.u. Trieste.

39 C. MARCATO, s.u. Oderzo.

40 G.B. PELLEGRINI - A.L. PROSDOCIMI, La lingua venetica, Padova, 1967. I. Le iscrizioni, a cura di G.B. PELLEGRINI - A.L. PROSDOCIMI, p. 602.

41 L'iscrizione, rinvenuta nella Pannonia Superior, suona P. DOMATIUS P. [F.] TERGITIO NEGOTIATUR (CIL, III, 4251). 
Per quanto riguarda l'origine del toponimo, c'è chi assegna Tergeste al venetico ${ }^{43} \mathrm{e}$ chi all'illirico ${ }^{44}$. L'opzione non è facile, anche perché la conoscenza dell'illirico è quella che è. Noi, comunque, propendiamo per la seconda ipotesi, perché riteniamo illirici sia il tema ${ }^{*}$ terg-, sia il suffisso $-s t^{45}$ cui si aggiunge l'accertata presenza di un elemento illirico nella toponomastica istriana ${ }^{46}$. Da Tergeste, concordemente, non si possono separare né Opitergium, né Tergolape 47 .

\section{Povzetek \\ OPOMBE K ITALIJANSKI TOPONOMASTIKI, I}

Obravnava se slovar krajevnih imen v Italiji (Dizionario di toponomastica. Storia e significato dei nomi geografici italiani, Torino 1990), pomembno delo, saj Italija tako celostnega pregleda krajevnih imen $\mathrm{z}$ vsem znanstvenim aparatom še ni imela, medtem ko so bila osebna in rodbinska imena že zelo dobro obdelana. Avtor pa vendarle kritično tehta nekaj razlag, zlasti etimoloških, dodaja pomembne podrobnosti ali predlaga drugačno razlago vira. Posebej je zanimivih nekaj toponimov iz grškega jezikovnega vpliva (Palermo, Trapani, Teramo/Terni), iz severne Italije (Aosta, Sesto Calende), za nas pa seveda latinsko krajevno ime TERGESTE. Ni dvoma, da je trg v slovanskih jezikih istega izvora. Enak vir kot Trst kaže še lat. krajevno ime OPITERGIUM (danes Oderzo v Benečiji). Če ni mogoče dvomiti o pomenu osnove terg, pa med etimologi ni soglasja, ali naj bi bil izraz venetski ali ilirski. Avtorju se zdi ilirski vir verjetnejši, tudi zaradi pogostnih ilirskih jezikovnih prvin v istrski toponomastiki.

$\overline{42}$ Questa conclusione è rifiutata senza fondati motivi, come giustamente rilevano Aldo ILuigi Prosdocimi e Giovan Battista Pellegrini (G.B. PELLEGRINI - A.L. PROSDOCIMI, Op.cit., rispettivamente alle pp. 27 e 602) da Michel Lejeune (M. LEJEUNE, Les inscriptions vénètes, Trieste - Udine, 1965, p. 11 n. 31). E forse la 'prudenza' dell'Autrice delle voci si spiega con l'autorevolezza dello studioso transalpino.

43 G.B. PELLEGRINI - A.L. PROSDOCIMI, Op.cit., pp. 601-602; M. DORIA, Toponomastica preromana dell'Alto Adriatico, in Antichità Altoadriatiche, Udine, 1972, II, pp. 17-42; G. FOGOLARI - A.L. PROSDOCIMI, I veneti antichi. Lingua e cultura, Padova, 1988 [non 1987 come compare nella voce], pp. 397-401.

44 G. MEYER, Etymologisches, "IF", I (1892), pp. 319-329 [Der Stadtname Triest, pp. 323-324]; H. KRAHE, Die alten balkanillyrischen geographischen Namen, Heidelberg, 1925, p. 109; G. BONFANTE, Trieste e l'Italia, "Intervento", 38-39 (1979), pp. 1-4; G. BONFANTE, Il nome di Trieste, "Lincei-Rendiconti", XLII/1-2 (1987) (ma 1988), pp. 11-12.

45 H. KRAHE, Op.cit., pp. 68 segg.; G. BONFANTE, Il nome di Trieste cit., p. 11.

46 M. DORIA, Art.cit., pp. 30-32.

47 Città del Norico, regione notoriamente abitata anche da Xlliri. 